\title{
Influence of temporal masking on click-pair discriminability
}

\author{
LAWRENCE L. FETH and HONOR O'MALLEY \\ Department of Audiology and Speech Sciences, Purdue University, West Lafayette, Indiana 47907
}

\begin{abstract}
When temporal auditory acuity is estimated using time-reversed click pairs, the asymmetry of temporal masking may influence the results. To investigate this hypothesis, we first established normal-hearing listeners' psychometric functions for the discrimination of timereversed click pairs. An attempt to relate listeners performances in click-pair discrimination to their performances in forward and backward masking tasks was unsuccessful, probably due to the presence of spectral artifact in the temporal masking paradigms. We then investigated listeners' abilities to discriminate time-reversed click pairs in which the intensity of the lower level click in one pair was altered. This alteration was introduced at random in order to minimize the influence of spectral artifact. Our results suggest that the course of temporal masking influenced the discriminability of the modified click pairs. Thus, the discrimination of timereversed click pairs may yield a biased estimate of auditory temporal acuity.
\end{abstract}

It has been suggested that a listener's ability to discriminate between time-reversed transient signals may be regarded as an estimate of the ear's temporal acuity (Green, 1971, 1973; Patterson \& Green, 1970; Wier \& Green, 1975). In particular, Ronken (1970) demonstrated that listeners with normal hearing were able to distinguish one transient composed of two clicks of unequal amplitude separated by a brief interval of time $(\Delta t)$ from a transient which presented those same clicks in reverse temporal order. Figure 1a illustrates these signals.

The signal labeled FORWARD is composed of two very brief $(100-\mu \mathrm{sec})$ clicks which occur with a temporal separation of $\Delta t$. In this signal, the amplitude of the first click is greater than that of the second by an amount $\Delta I$. When these same two clicks are presented in reversed temporal order, the BACKWARD transient results. Ronken demonstrated that the energy-density spectra calculated for the FORWARD transient and the BACKWARD transient are equivalent for equal $\Delta I$ and $\Delta t$ values within the two signals. The signals differ only in their phase spectra, which reflect the order of events in a click pair.

Ronken reported the just discriminable $\Delta \mathrm{t}$ to be $2 \mathrm{msec}$, a temporal threshold estimate supported by several related studies (Green, 1971). It is thought

This work was supported by the Department of Audiology and Speech Sciences, Purdue University. Preparation of the manuscript was supported, in part, by a grant from the National Institutes of Health. The authors gratefully acknowledge the contributions of S. B. Resnick to the design of this study and the discussion of results, and thank David M. Green for his critical reading of an earlier version of this paper. Portions of this work were reported at the Spring 1975 meeting of the Acoustical Society of America. that such time-reversed transient signals are suited to the study of temporal acuity because they avoid the problem of spectral confounding described by Leshowitz (1971). Leshowitz has shown that auditory temporal acuity can be drastically overestimated when spectral artifacts are available for wellpracticed listeners to use in making their discriminations.

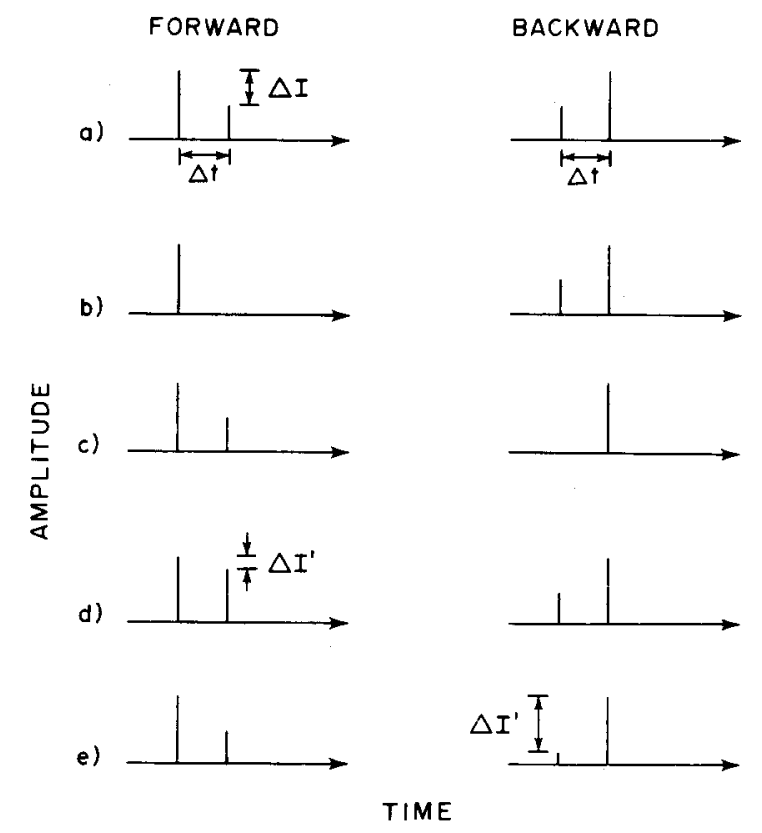

Figure 1. Schematic representation of stimulus configurations. (a) Standard time-reversed click pairs used in Experiment 1. (b) and (c) "Probe" click and "masker" clicks used in Experiment 2. (d) and (e) Modified click pairs used in Experiment 3. The columns are labeled FORWARD and BACKWARD. respectively, to suggest the temporal order of the clicks in the corresponding temporal masking paradigm. 
Green (1971) indicated that the temporal separation, $\Delta t$, at which these time-reversed transients became just discriminable could be taken as an estimate of the listener's auditory temporal acuity. He suggested that when $\Delta t$ was less than the ear's minimum time constant, the time-reversed signals would be indiscriminable because each presents the same distribution of broad-band energy over time. With $\Delta t$ values greater than the minimum time constant, each click in the two-click pair would represent a unitary auditory event. The time-reversed pairs should then be discriminable because the listener could determine that the sequence of events differed in the two presentations. The value of $\Delta t$ at which the time-reversed transients were just discriminable might then yield an estimate of the listener's temporal acuity.

Resnick and Feth (1975a, 1975b) challenged the interpretation of the results of experiments employing time-reversed click pairs. They found that altering both the intensity relations within the click pairs $(\Delta \mathrm{I})$ and the overall intensity of the signal complex affected the discriminability of these transients. When Resnick and Feth presented the click pairs at 45-dB sensation level (SL), the temporal separation, $\Delta t$, at which the signals became discriminable increased systematically from a value less than $0.5 \mathrm{msec}$ to $1.8 \mathrm{msec}$ as the intensity difference between the clicks in a pair increased from 3 to $24 \mathrm{~dB}$. At $75 \mathrm{~dB} \mathrm{SL}$, the relationship between just discriminable $\Delta t$ and the value of $\Delta I$ was reversed. Resnick and Feth suggested that, rather than reflecting only the influence of auditory temporal processing, listener performance was mediated by the masking of the lower level click by the higher level click.

Where the higher level click is first, forward masking may occur. In the reverse temporal order, backward masking may occur. At a given temporal separation, $\Delta t$, the effectiveness of the higher level click as a masker may differ for forward and backward masking conditions. This differential effect is often called the asymmetry of temporal masking. Resnick and Feth argued that the discriminability of timereversed click pairs was good when the lower level click was detectable in one pair, but remained masked in the other pair. Most often, the lower level click was detectable in the BACKWARD pair, but undetectable in the FORWARD pair. However, this argument was not tested experimentally.

In order to test directly the hypothesis that the asymmetry of temporal masking determines listener performance in the discrimination of time-reversed click pairs, we first gathered normative data for five listeners in that task (Experiment 1). We then compared their performance in the simple click-pair discrimination task with their performance in a rather complicated intensity-titration experiment
(Experiment 3). Finally, for three of the five original listeners, we attempted to relate performance in simple forward or backward masking experiments (Experiment 2) to their performance in time-reversed click pair discrimination.

\section{EXPERIMENT 1: DISCRIMINATION OF TIME-REVERSED CLICK PAIRS}

\section{Method}

Subjects. Five normal-hearing young adults served as subjects. Their thresholds were no poorer than $15 \mathrm{~dB}$ re the ANSI (1969) standard for frequencies at octave intervals from 250 to $8,000 \mathrm{~Hz}$. Three subjects were undergraduates at Purdue University who were paid an hourly wage for their services in the experiment. (They received approximately 40,000 practice trials in discriminating time-reversed click pairs before the data reported here were collected.) The other two subjects were the authors, one of whom (S3) had served as a listener in the Resnick and Feth (1975b) study.

Apparatus. All of the signals used in this study were generated by a digital-to-analog converter interfaced to a PDP-8/L computer. Generation, timing, and presentation of the signals in a standard two-interval forced choice (2IFC) paradigm were controlled by the computer. Signals were delivered to the listeners through 10- $\Omega$ TDH-39 headphones mounted in MX-41/AR cushions.

The level of the more intense click was fixed at a peak equivalent sound pressure level (SPL) of $75 \mathrm{~dB}$. Peak equilvalent SPL is that sound pressure level generated in a $6-\mathrm{cc}$ coupler by a $1,000-\mathrm{Hz}$ tone, the negative peak amplitude of which equals the first negative peak of the click. This level was approximately $45 \mathrm{~dB}$ above the averaged thresholds of the three undergraduate listeners. The relative intensity, $\Delta \mathrm{I}$, of the less intense click was $-3,-6$, -12 , or $-24 \mathrm{~dB}$. Interclick intervals, $\Delta \mathrm{t}$, were specified at 0.5 , $0.75,1.0,1.5,2.0,2.5$, and $5.0 \mathrm{msec}$.

Procedures. Subjects were asked to discriminate between the time-reversed click-pair signals displayed in Figure 1a. A standard 2IFC procedure with feedback was employed. A randomization scheme was used to select the combination of $\Delta t$ and $\Delta I$ values to be tested in blocks of 50 trials each. Although the listeners ran one, two, or three at a time, the $\Delta t-\Delta I$ combinations were presented according to the same randomization scheme.

\section{Results}

The psychometric functions for the discrimination of time-reversed click pairs are presented in Figure 2. Each graph represents the percentage of correct discriminations, $P(C)$, in the $2 \mathrm{IFC}$ task as a function of $\Delta t$ for one subject. A separate function is drawn for each value of $\Delta \mathrm{I}$. Each point represents at least 200 observations for the three undergraduate listeners (S1, S2, S4); 100 observations per point were collected for the authors (S3, S5). The wide range of individual performance found by Resnick and Feth (1975b) is apparent here. The performance of the listener (S3) who participated in the Resnick and Feth study is consistent with that subject's performance in the earlier study. The performance of listener S5 is more like that of Resnick and Feth's listeners at the higher signal level (75 dB SL), perhaps because S5's hearing threshold was 10 to $15 \mathrm{~dB}$ better than the 

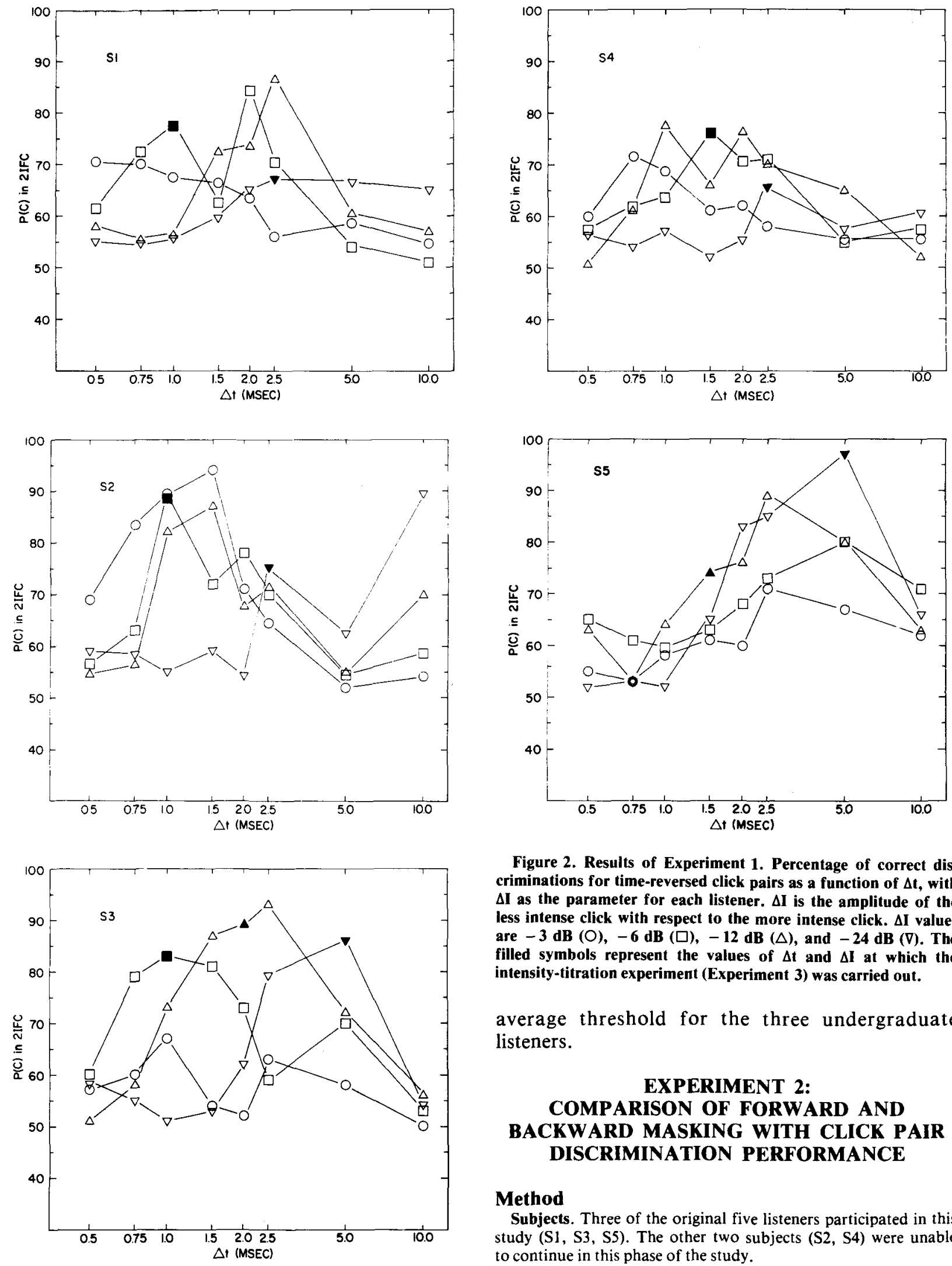

Figure 2. Results of Experiment 1. Percentage of correct discriminations for time-reversed click pairs as a function of $\Delta t$, with $\Delta I$ as the parameter for each listener. $\Delta I$ is the amplitude of the less intense click with respect to the more intense click. $\Delta I$ values are $-3 \mathrm{~dB}(O),-6 \mathrm{~dB}(\square),-12 \mathrm{~dB}(\Delta)$, and $-24 \mathrm{~dB}(\nabla)$. The filled symbols represent the values of $\Delta t$ and $\Delta I$ at which the intensity-titration experiment (Experiment 3) was carried out.

average threshold for the three undergraduate listeners.

\section{EXPERIMENT 2: COMPARISON OF FORWARD AND BACKWARD MASKING WITH CLICK PAIR DISCRIMINATION PERFORMANCE}

\section{Method}

Subjects. Three of the original five listeners participated in this study $(\mathrm{S} 1, \mathrm{~S} 3, \mathrm{~S} 5)$. The other two subjects (S2, S4) were unable to continue in this phase of the study. 
Procedures. Although these data were actually collected after those to be reported as Experiment 3, it seems instructive to discuss them here. Experiment 2 used essentially the same apparatus and procedures described earlier. Overall signal level was again at $45 \mathrm{~dB} \mathrm{SL}$; the $\Delta \mathrm{I}$ values ranged from -3 to $-45 \mathrm{~dB}$ in $3-\mathrm{dB}$ steps. The values of $\Delta t$ were $1.0,1.5,2.0,2.5$, and $5.0 \mathrm{msec}$. The click stimuli, however, were different. Figure $1 \mathbf{b}$ indicates that the lower level click can be deleted from the FORWARD transient to produce a backward masking experiment. In a similar fashion, the lower level click can be deleted from the BACKWARD transient to produce a forward masking paradigm (Figure 1c). In either case, the remaining smaller click becomes the "probe" to be detected in the presence of the larger, masking click.

In order to generate temporal masking functions, both forward and backward masking experiments were run using the signals described above. The subjects were required to indicate which listening interval contained the probe click. Timing and feedback for the 2IFC paradigm were identical to that used in Experiment 1.

\section{Results}

Temporal masking functions are shown in Figure 3. Each panel presents the relative probe level $(\Delta \mathrm{I}$ in Figure $1 \mathrm{~b}$ or Figure $1 \mathrm{c})$ for which the listener achieved $75 \%$ correct detections in a 2 IFC procedure with $\Delta t$ set to the value shown on the abscissa. These $75 \%$ points were interpolated from each listener's psychometric functions for detection of the probe in either the forward or backward masking task. In Figure 3 , the results for backward masking are plotted to the left of the ordinate, with $\Delta t$ values shown as negative numbers. For each listener, at least 200 observations, at each of two or three values of $\Delta \mathbf{l}$ for each $\Delta t$ were used to generate the detection functions. The error bars for each datum represent interpolated $95 \%$ and $55 \%$ points from the detection functions. Note that there are no error bars about the datum for $\mathrm{S} 5$ in the backward masking condition where $\Delta \mathrm{t}=-5.0 \mathrm{msec}$. This point does not represent S5's threshold for that condition; rather, it indicates that subject's performance at the limit of the dynamic range of the $D / A$ converter.

The results for each observer appear to show the asymmetry of temporal masking which Resnick and Feth thought might mediate time-reversed click pair discrimination. That is, for a given magnitude of $\Delta t$, there is more masking evident when the probe follows the masker (forward masking) than when it precedes it (backward masking). This was as expected and has been reported previously (Babkoff \& Sutton, 1968; Raab, 1961). Ronken (1970) had also presented temporal masking results for one of his listeners and found no relationship between the asymmetry of temporal masking and his time-reversed click-pair discrimination results.

Table 1 presents comparisons of performances in the forward and backward masking tasks with performances in click-pair discrimination for the three listeners who participated in Experiment 2. To review, recall that Resnick and Feth (1975b) proposed that discrimination performance should be
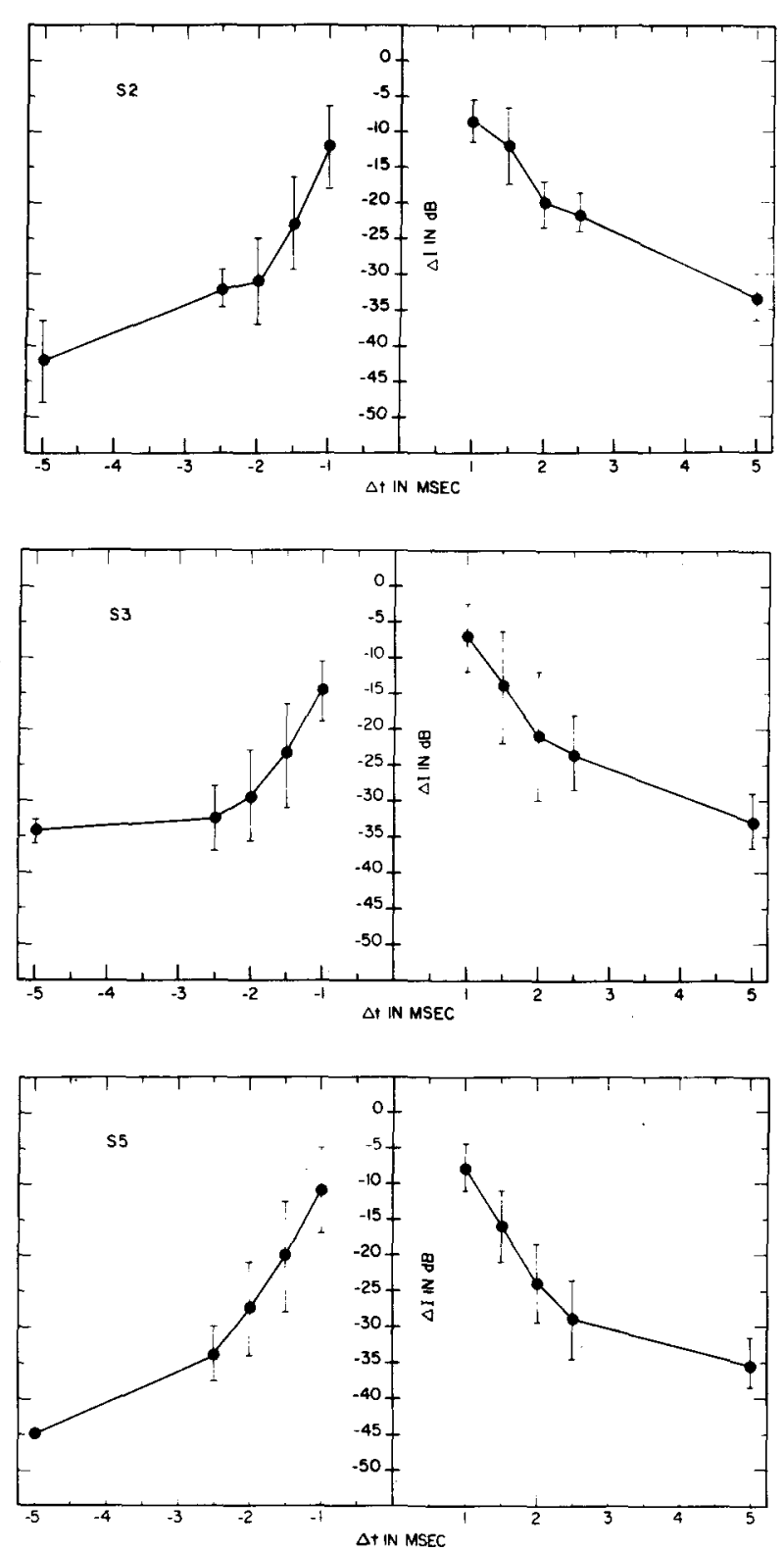

Figure 3. Temporal masking functions for the three listeners in Experiment 2. Each datum represents the relative level of the probe click at which the listener's percentage of correct detections was 75 in a 2IFC task. The $75 \%$ point was interpolated from straight-line approximations of each listener's psychometric functions. The error bars represent interpolated values of $95 \%$ and $55 \%$. Forward masking results are plotted to the right of the ordinate; backward masking results are plotted to the left, at negative values of $\Delta t$.

good when the lower level click was masked by the larger click in one transient pair, but detectable in the time-reversed transient pair. Thus, click-pair discrimination performance should approach $100 \%$ for a given $\Delta t-\Delta I$ combination if the probe is above threshold in the BACKWARD transient, but below threshold in the FORWARD transient. In Table 1, 
Table 1

Comparison of Listeners' Performances in Forward and Backward Masking Experiments With Their Performances in Click-Pair Discrimination Under the Same Signal Conditions

\begin{tabular}{|c|c|c|c|c|c|}
\hline \multirow[b]{3}{*}{ Listener } & \multirow{2}{*}{\multicolumn{2}{|c|}{ Condition }} & \multicolumn{3}{|c|}{$\mathrm{P}(\mathrm{C})$} \\
\hline & & & \multirow[b]{2}{*}{ Forward } & \multirow[b]{2}{*}{ Backward } & \multirow{2}{*}{$\begin{array}{l}\text { Discrimi- } \\
\text { nation }\end{array}$} \\
\hline & $\Delta \mathrm{t}(\mathrm{msec})$ & $\Delta \mathrm{I}(\mathrm{dB})$ & & & \\
\hline
\end{tabular}

\begin{tabular}{|c|c|c|c|c|c|}
\hline \multicolumn{6}{|c|}{ Dissimilar } \\
\hline S2 & $\begin{array}{l}1 \\
1.5 \\
2.5\end{array}$ & $\begin{array}{l}-12 \\
-12 \\
-24\end{array}$ & $\begin{array}{l}52 \\
71 \\
56\end{array}$ & $\begin{array}{l}73 \\
99 \\
95\end{array}$ & $\begin{array}{l}82 * \\
87^{*} \\
75^{*}\end{array}$ \\
\hline S3 & $\begin{array}{l}1 \\
1 \\
2.5\end{array}$ & $\begin{array}{l}-6 \\
-12 \\
-24\end{array}$ & $\begin{array}{l}78 \\
55 \\
69\end{array}$ & $\begin{array}{l}96 \\
88 \\
96\end{array}$ & $\begin{array}{l}83^{*} \\
73^{*} \\
79^{*}\end{array}$ \\
\hline S5 & 1 & -12 & 51 & 72 & 64 \\
\hline \multicolumn{6}{|c|}{ Similar } \\
\hline S2 & $\begin{array}{l}1 \\
1 \\
1.5 \\
2 \\
2 \\
2 \\
5 \\
5 \\
5 \\
5\end{array}$ & $\begin{array}{l}-6 \\
-24 \\
-3 \\
-3 \\
-6 \\
-12 \\
-3 \\
-6 \\
-12 \\
-24\end{array}$ & $\begin{array}{r}93 \\
\sim 50 \\
98 \\
100 \\
100 \\
99 \\
100 \\
100 \\
100 \\
100\end{array}$ & $\begin{array}{r}97 \\
\sim 50 \\
100 \\
100 \\
100 \\
100 \\
100 \\
100 \\
100 \\
100\end{array}$ & $\begin{array}{l}89 \\
55^{*} \\
94 \\
71 \\
78 \\
68 \\
52^{*} \\
54^{*} \\
55^{*} \\
63^{*}\end{array}$ \\
\hline S3 & $\begin{array}{l}1 \\
2 \\
2 \\
2 \\
2.5 \\
2.5 \\
5\end{array}$ & $\begin{array}{r}-24 \\
-3 \\
-6 \\
-12 \\
-6 \\
-12 \\
-24\end{array}$ & $\begin{array}{r}\sim 50 \\
100 \\
100 \\
96 \\
100 \\
100 \\
98\end{array}$ & $\begin{array}{r}51 \\
100 \\
100 \\
99 \\
100 \\
100 \\
100\end{array}$ & $\begin{array}{l}51^{*} \\
52^{*} \\
73 \\
89 \\
59^{*} \\
93 \\
86\end{array}$ \\
\hline S5 & $\begin{array}{l}1 \\
1 \\
1 \\
2 \\
2.5 \\
2.5 \\
5 \\
5 \\
5\end{array}$ & $\begin{array}{l}-3 \\
-6 \\
-24 \\
-12 \\
-3 \\
-12 \\
-6 \\
-12 \\
-24\end{array}$ & $\begin{array}{r}97 \\
89 \\
\sim 50 \\
99 \\
100 \\
100 \\
100 \\
100 \\
100\end{array}$ & $\begin{array}{r}100 \\
93 \\
61 \\
100 \\
100 \\
100 \\
100 \\
100 \\
100\end{array}$ & $\begin{array}{l}58^{*} \\
60^{*} \\
52^{*} \\
76 \\
71 \\
89 \\
80 \\
80 \\
97\end{array}$ \\
\hline
\end{tabular}

Note-Those entries which tend to support the suggested influence of the asymmetry of temporal masking on click-pair discrimination are marked by an asierisk (*).

we characterize such performance as being DISSIMILAR in forward and backward masking. Of the seven entries which show dissimilar detection performance in forward and backward masking, six result in click-pair discrimination performance, $P(C)$, of approximately 75 or greater. These six entries, marked by an asterisk $\left({ }^{*}\right)$ may be interpreted as supporting the proposed role of the asymmetry of temporal masking in the discrimination of timereversed click pairs. For listener S5 at $\Delta \mathrm{t}=1 \mathrm{msec}$ and $\Delta \mathrm{I}=-12 \mathrm{~dB}$, detection performance is at chance $(51 \%)$ in forward masking and approaches the just detectable level $(71 \%)$ in backward masking.
In click-pair discrimination, however, S5 attains a $P(C)$ of 64 .

If the asymmetry of temporal masking were the determinant of click-pair discriminability, then discrimination performance should fall to chance levels whenever detection performance is uniformly good (approaching 100\%) or bad (approaching 50\%) in both forward and backward masking. Such performance by our listeners is labeled SIMILAR in Table 1. Of the 26 entries in which a listener's detection performance in forward masking was similar to that listener's performance in the comparable backward masking task, 11 support the hypothesis that the asymmetry of temporal masking mediates discrimination performance. These entries are also marked by an asterisk (*). For those entries which do not reflect the influence of the asymmetry of temporal masking, discrimination scores ranges from just discriminable levels to almost perfect discriminability. Clearly, since only half of the entries in Table 1 can be interpreted as supporting the temporal asymmetry explanation, the listeners must be sensitive to some additional aspect of the stimuli.

The failure to demonstrate directly the relationship between the asymmetry of forward and backward masking and listener performance in the discrimination of time-reversed click pairs should not be interpreted as evidence that this asymmetry has no bearing on listener performance. Recall that wellpracticed listeners are quite good at detecting subtle spectral differences between transient signals (Leshowitz, 1971). In conducting this direct test, we had ignored this consideration. The energy-density spectrum for a masking click alone has the familiar $|\sin \mathrm{x} / \mathrm{x}|$ shape, but adding a probe click at a small delay, $\Delta t$, introduces ripples into the spectrum (Ronken, 1970). The ripples are periodic in frequency with spacing inversely proportional to $\Delta t$. The depth of these spectral ripples depends upon the relative magnitudes of the clicks in a pair. There is a maximum peak-to-valley ratio when the clicks are equal in amplitude; the ratio becomes smaller as the disparity in amplitude increases.

For a given $\Delta \mathrm{t}-\Delta \mathrm{I}$ combination, the location of spectral peaks and valleys and the peak-to-valley intensity ratio are identical for both forward and backward masking paradigms. If the artifact alone determined performance, we would expect symmetrical temporal masking functions. Since the asymmetry of temporal masking was evident in the data shown in Figure 3, the saliency of the spectral cue may have varied with the paradigm. In addition, we might expect large individual differences in the temporal masking results if the saliency of the spectral artifact varied for both $\Delta t$ and probe level. This was the case with our data. 


\section{EXPERIMENT 3: INTENSITY-TITRATION}

In order to demonstrate the influence of temporal masking on the discriminability of time-reversed transient signals, two alternatives are available. One could alter the relative intensity $(\Delta \mathrm{l})$ between the clicks in one pair, or one could separate the clicks in a transient by a greater $\Delta t$ than in its time-reversed counterpart. If the latter alternative were chosen, however, the temporal alteration would change the loci of spectral peaks and valleys for the altered pair, resulting in a predictable change in the pitch of that signal (Bilsen, 1966). Pitch differences between the transients would lead to gross overestimation of auditory temporal resolving powers.

We, instead, returned to the original paradigm illustrated in Figure la. If, for a given temporal separation and relative intensity, there were an asymmetrical masking effect, then one might alter the relative intensity of the clicks in only one transient. The asymmetry of temporal masking led us to predict that the more intense click would yield greater masking of the less intense click in the FORWARD transient rather than in the BACKWARD transient. For example, if the lower level click is thought to be undetectable in the FORWARD transient, its level might be increased, as in Figure 1d, until it is equal in detectability with the unaltered click in the BACKWARD transient. If discriminability of the FORWARD and BACKWARD transients depends upon the detectability of the lower level click in the BACKWARD pair, then the alteration just described should degrade a listener's performance in the discrimination task. Rather than increase the level of the smaller click in the FORWARD transient, we could as well decrease its level in the BACKWARD case (Figure 1e). This too should lead to poorer discriminability of time-reversed transients.

In altering the relative intensity between the clicks in one pair, the spectral peaks and valleys do not move. This alteration simply changes the ratio of the amplitude at a peak to that at a valley, thus perhaps altering the strength of the pitch, but not the pitch itself. We are still faced with the problem that alteration of the click level in only one of the two transients introduces a spectral artifact which well-practiced listeners might use in the discrimination task. Often, if one encounters an artifact which cannot be removed from the signal, a randomization scheme may be devised such that the artifact is rendered an unreliable cue upon which to base responses (Henning, 1966; Leshowitz, 1971). Thus, in each block of the experiment in which the discriminability of timereversed click pairs was being determined, we chose to introduce an intensity modification on only $20 \%$ of the trials. If a listener's ability to discriminate between the unaltered transients is good, perhaps at $70 \%$ correct, and if it were mediated by asymmetrical temporal masking of the lower level click, then a listener's performance on those trials in which the intensity was altered should be degraded. If a listener is able to use the spectral difference, it should result in improved performance on the altered trials.

\section{Method}

As before, the laboratory computer controlled the sequence of events within trials and the generation and presentation of the test signals. Click-pair discriminability was again assessed using a 2IFC procedure with feedback. Overall signal level was $45 \mathrm{~dB}$ SL. The $\Delta \mathrm{t}-\Delta \mathrm{I}$ combinations used for each subject in Experiment 3 were selected on the basis of individual subject performance in the discrimination of standard time-reversed click pairs (Experiment 1). The subjects' task was to identify the interval in which the backward transient occurred.

From the psychometric functions generated by each of the five original subjects at every $\Delta t-\Delta I$ combination of the standard transient pairs, we determined those combinations where $P(C)$ was approximately 70 . Modifications of the less intense click were made only where a subject met this criterion. These $\Delta t-$ $\Delta \mathrm{I}$ combinations for each subject are illustrated in Figure 2 as filled symbols. Wherever possible, an effort was made to test subjects at the same combination of $\Delta t-\Delta I$ values.

The effects of intensity modification of the less intense click were explored separately in both the FORWARD and the BACKWARD transients. On approximately $80 \%$ of the experimental trials, $\Delta \mathrm{I}$ in both transients was identical to that in the standard condition (Figure 1a). At random, on about $20 \%$ of the 50 trials in each experimental block, the level of the less intense click was altered in only one of the transients. The intensity modification was made either by increasing the level of the less intense click in the FORWARD transient (Figure 1d) or by decreasing the level of the less intense click in the BACKWARD transient (Figure le). The relative intensity of the modified transient, $\Delta \mathrm{I}^{\prime}$, was always set equal to one of the three remaining values. This restricted the value which the less intense click could assume in the modified FORWARD or BACKWARD transient and was dependent upon the specified $\Delta \mathrm{I}$ value of the standard transient pairs in a given experimental block. For example, when $\Delta \mathrm{I}=-6 \mathrm{~dB}$ in the standard transient pairs, $\Delta \mathrm{I}$ ' could equal -12 or $-24 \mathrm{~dB}$ in the modified BACKWARD transient. When $\Delta \mathrm{I}=-6 \mathrm{~dB}$, similarly, the value of the modified FORWARD transient was limited to $-3 \mathrm{~dB}$. Obviously, when $\Delta \mathrm{I}=-3 \mathrm{~dB}$, no modification of the FORWARD transient was possible. Likewise, the level of the lower level click was not reduced further when $\Delta \mathrm{I}=-24 \mathrm{~dB}$ in the BACKWARD transient.

For most $\Delta \mathrm{t}-\Delta \mathrm{l}$ combinations of the standard transient pairs where a subject met the $P(C)=70$ criterion, the experimental intensity modifications were introduced over 10 blocks of 50 trials each. $P(C)$ values were tabulated separately for the approximately 400 trials in which the transients were unmodified, and for the remaining 100 trials in which a given intensity modification was introduced. It was observed that $P(C)$ values over the 400 unmodified transient trials generally did not differ from those obtained when only the standard transients were presented on all trials. No more than 100 trials per day overall contributed to the determination of either $P(C)$ for a given $\Delta t-\Delta I-\Delta I^{\prime}$ condition.

\section{Results}

The effects of the intensity modifications on subject performance are displayed in Figure 4. The height of each bar represents the $P(C)$ value for a given experimental condition. The unfilled portion of 
the bar represents the $P(C)$ for the standard pair. The diagonally striped portion indicates $P(C)$ for the modified BACKWARD transient pairs; the shaded portion indicates $P(C)$ for the modified FORWARD transient pairs. Keep in mind that an increase in the magnitude of the relative intensity from $\Delta \mathrm{I}$ to $\Delta \mathrm{I}$ represents a decrease in the level of the probe click.

When $\Delta \mathrm{I}=-6 \mathrm{~dB}$, three subjects achieved criterion performance at $\Delta t=1 \mathrm{msec}$, while one subject met criterion at $\Delta t=1.5 \mathrm{msec}$. In Figure $4 \mathrm{a}$, the two rightmost bars for each subject represent the results of the modification of the BACKWARD transient. The leftmost bar for each subject depicts the results of the modification of the FORWARD transient. In all cases, whether the modification of the FORWARD or the BACKWARD transient was under test, subjects' discriminability of the standard transients remained high and relatively unchanged. When the BACKWARD transient was modified, however, discriminability was always reduced. In fact, no subject obtained $P(C)$ higher than 60 when $\Delta \mathrm{I}^{\prime}$ was set to $-24 \mathrm{~dB}$; S2's $\mathrm{P}(\mathrm{C})$ decreased to 35 under this condition. Examination of Figure 4a would suggest that when $\Delta \mathrm{I}=-6 \mathrm{~dB}$ and the FORWARD transient is modified, listener performance is unaffected. Two subjects show an insignificant decrement in $P(C)$. One subject exhibits a slight improvement in performance over the standard condition, while the performance of the remaining subject is equivalent for both the standard and the modified trials.

Figure $4 \mathrm{~b}$ shows the iesults for two subjects who met criterion when $\Delta \mathrm{I}=-12 \mathrm{~dB}$ and $\Delta \mathrm{t}=2 \mathrm{msec}$ or $1.5 \mathrm{msec}$, respectively. In this figure, for each subject, the BACKWARD transient modification result is illustrated in the rightmost bar, while the FORWARD transient modification results are pictured in the two leftmost bars. Again, the discriminability of the standard transients is comparable to that demonstrated by each subject in the preliminary testing. At $\Delta \mathrm{I}=-12 \mathrm{~dB}$, however, modification of either the FORWARD or the BACKWARD transient results in a marked reduction in $P(C)$ for both subjects.

For the $\Delta \mathrm{I}=-24 \mathrm{~dB}$ pairs, the restriction placed on the values of $\Delta I^{\prime}$ permitted examination only of the effects of modifying the FORWARD transient. Data were gathered for three subjects at $\Delta \mathrm{t}=$ $2.5 \mathrm{msec}$ (Figure 4c) and for two subjects at $\Delta \mathrm{t}=$ $5.0 \mathrm{msec}$ (Figure 4d). We have previously noted that when the modified trials were introduced, subject performance for the standard click pairs generally remained unaffected as compared with the results of Experiment 1. When the $\Delta t-\Delta \mathrm{I}$ combination $2.5 \mathrm{msec} /-24 \mathrm{~dB}$ was presented in the intensitytitration experiment (Figure 4c), however, S1 and S4 showed improvements over their performances displayed as filled symbols in Figures $2 \mathrm{a}$ and $2 \mathrm{~d}$, respectively. The performances of both subjects im-
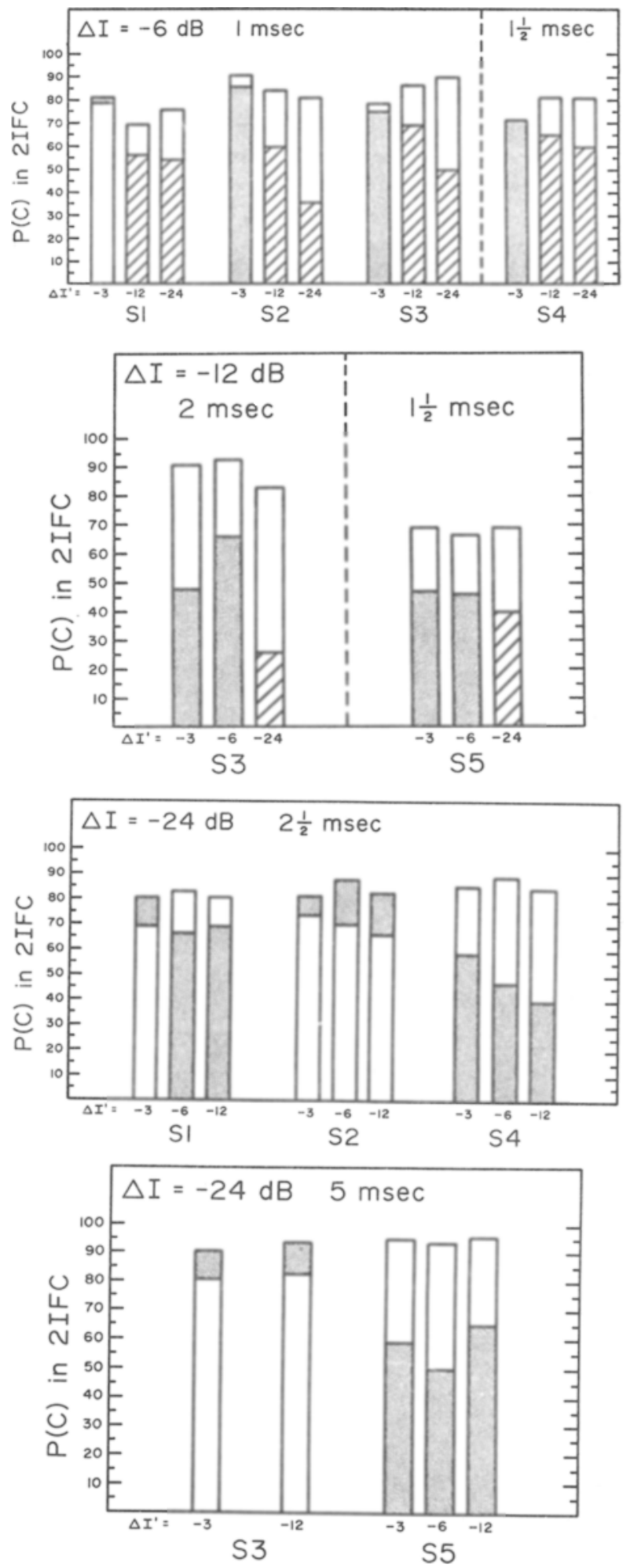

Figure 4. Individual listener performance for discrimination of time-reversed click pairs. The unfilled portion of each bar represents $P(C)$ for discrimination of standard time-reversed click pairs. The striped portion indicates $P(C)$ for those trials on which the BACKWARD transient was modified. The shaded portion represents $P(C)$ for those trials on which the FORWARD transient was modified. The values of $\Delta I$ and $\Delta t$ are indicated at the top of each figure. $\Delta I^{\prime}$ values are indicated beneath each bar. 
proved from approximately $68 \%$ to over $80 \%$ correct. Unlike the other three subjects, S1 and S4's psychometric functions for the discrimination of time-reversed click pairs rarely exhibited $P(C) s$ above 80. We attribute their improved discrimination of standard time-reversed click pairs in the intensitytitration experiment to increased practice in the task.

When $\Delta \mathrm{I}=-24 \mathrm{~dB}, \mathrm{~S} 4$ and $\mathrm{S} 5$ showed a marked decrease in $P(C)$ when the magnitude of $\Delta I^{\prime}$ was reduced in the FORWARD transient. S2 and S3 showed the opposite effect, an improvement in performance, as a consequence of the reduction in the magnitude of $\Delta \mathrm{I}^{\prime}$. The performance of $\mathrm{S} 1$ showed both effects: $\mathrm{P}(\mathrm{C})$ declined slightly when $\Delta \mathrm{I}^{\prime}=-12$ or $-6 \mathrm{~dB}$ in the FORWARD transient, but increased when $\Delta \mathrm{I}^{\prime}$ was changed to $-3 \mathrm{~dB}$.

In summary, the results indicate that reduction in the level of the less intense click in the BACKWARD transient produced a consistent decrease in transient discriminability. The effect of increasing the level of the less intense click in the FORWARD transient was less consistent and appeared to depend upon the value of $\Delta \mathbf{l}^{\prime}$.

\section{DISCUSSION}

Discriminability of time-reversed click pairs was proposed as a means of measuring auditory temporal acuity because the signals are free of spectral differences. Spectral differences might lead to an overestimation of the temporal resolving power of the ear, if listeners used them in the forced choice discrimination task. Resnick and Feth (1975b) suggested that the discriminability of time-reversed click pairs was mediated by the asymmetry of temporal masking after finding that click-pair discriminability depended both upon the level of presentation and the relative intensity between the clicks in a pair.

This study was designed to demonstrate directly the dependence of click-pair discriminability upon the asymmetry of temporal masking. However, both attempts to demonstrate the role of temporal asymmetry in click pair discriminability necessitated the introduction of slight differences in the energydensity spectra of the signals. These spectral differences apparently provided our listeners with a subtle cue which was often of use in the forced choice tasks.

Our attempt to make a direct comparison between a listener's performance in forward and backward masking tasks and performance in click-pair discrimination under the same signal conditions (Experiment 2) was confounded by the listener's ability to respond to subtle spectral cues. The masking functions displayed in Figure 3 show the influence of both temporal masking and the detection of spectral artifact. Had artifact alone governed performance, we would expect the forward and backward mask- ing functions to be symmetrical about $\Delta t=0$. For all three subjects, the forward masking results exhibit somewhat higher masked thresholds at a given value of $\Delta t$ than do the comparable backward masking functions. We may even speculate that of the three listeners, S5 was most sensitive to the spectral artifact, because the forward and backward masking functions are more nearly symmetrical for that listener, and S5's performance at $\Delta \mathrm{t}=-5 \mathrm{msec}$ exceeded the limits of the equipment. Recall that the ratio of spectral peaks to valleys is equal in FORWARD and BACKWARD transient pairs, so that the spectral cue is equivalent in either masking condition. Perhaps because of the increased sensitivity to the spectral cue, S5 demonstrates the weakest support for the role of the asymmetry of temporal masking in determining click-pair discriminability.

The results of the intensity-titration experiment (Experiment 3) demonstrate that the asymmetry of temporal masking does influence click-pair discriminability. The click pairs became discriminable at interclick intervals at once sufficient to permit detection of the less intense click in the BACKWARD transient and insufficient to permit its detection in the FORWARD transient. We considered it probable that the increased discriminability which occasionally resulted from modification of the FORWARD transient (see, for example, Figures $4 c$ and 4d) was due to the subjects' appreciation of spectral differences in the click pairs. Such an explanation would follow since the spectral ripple inherent in a transient click pair exhibits an even greater depth when the FORWARD transient is modified. We can only conclude that the asymmetry of temporal masking was responsible for the degradation of discriminability when the BACKWARD transients were modified. The intensity-titration experiment, with its randomized presentation of modified click pairs on $20 \%$ of the trials, was designed to minimize the spectral artifact problems encountered in the forward and backward masking paradigms. The intent of the titration procedure, however, was not to measure the relative influences of masking asymmetry and artifact. Indeed, given the present data, it is not possible to quantify the differential effects of the asymmetry of temporal masking and spectral artifact on listener performance.

In light of our experimental evidence, the minimum stimulus duration permitting discrimination of the time-reversed click pairs cannot be considered a "basic limit" of the auditory system in processing acoustic information. It is a measure which is critically dependent both on overall intensity and the relative intensity between the clicks in a pair. These intensity variables are reflected as individual differences in the time course of forward and backward masking. 


\section{REFERENCES}

American National Standards Institute. Specifications for audiometers. ANSI S3.6-1969. New York, 1969.

BabKoff, H., \& Sutton, S. Monaural temporal masking of transients. Journal of the Acoustical Society of America, 1968, 44. 1373-1378.

BILSEN. F. A. Repetition pitch: Monaural interaction of a sound with the repetition of the same, but phase shifted, sound. Acustica, 1966, 17, 295-300.

Green, D. M. Temporal auditory acuity. Psychological Review. 1971, 78, 540-551.

GreEN, D. M. Temporal acuity as a function of frequency. Journal of the Acoustical Society of A merica, 1973, 54, 373-379.

HeNNing, G. B. Frequency discrimination of random-amplitude tones. Journal of the Acoustical Society of America, 1966. 39. 336-339.

LESHOWITZ, B. Measurement of the two-click threshold. Journal of the Acoustical Society of America, 1971, 49, 462-466.

Patterson, J. H., \& Green, D. M. Discrimination of tran- sient signals having identical energy spectra. Journal of the Acoustical Society of America, 1970, 48, 894-904.

RAAB, D. H. Forward and backward masking between acoustic clicks. Journai of the Acoustical Society of America, 1961, 33, 137-139.

Resnick, S. B., \& Fetr, L. L. Discriminability of modified time-reversed click pairs. Journal of the Acoustical Society of America, 1975, 57, Supplement No. 1, S6 (A). (a)

Resnick, S. B., \& Feth, L. L. Discriminability of time-reversed click pairs: Intensity effects. Journal of the Acoustical Society of America, 1975, 57, 1493-1499. (b)

Ronken, D. A. Monaural detection of a phase difference between clicks. Journal of the Acoustical Society of America, 1970, 47. 1091-1099.

WiER. C. C.. \& Green, D. M. Temporal acuity as a function of frequency difference. Journal of the Acoustical Society of America, 1975, 57, 1512-1515.

(Received for publication December 24, 1976; revision accepted June 10, 1977.) 